\title{
Actitud ante la intención de donación de cerebros con fines de investigación en adultos mayores
}

\author{
Attitude towards the intention of brain donation \\ with research purposes in elder adults
}

\author{
Marlette González-Méndez,,*‡ Nuria Aguiñaga-Chiñas, ${ }^{\S}$ \\ Guillermo Cantú-Quintanilla* \\ * Centro Interdisciplinar de Bioética. Universidad Panamericana. \\ ₹ Centro de Salud Manuel Escontria. \\ $\S$ Escuela de Psicología Universidad Panamericana.
}

\begin{abstract}
RESUMEN
El objetivo de este estudio consiste en evaluar la intención de los adultos mayores frente a la intención de donación de cerebros en materia de investigación. Material y métodos: Estudio descriptivo, transversal, población: muestra de 51 sujetos que asisten al Centro de Salud Manuel Escontria, ubicado en la zona de San Ángel. Resultados: De los 46 sujetos incluidos en el estudio, 78.3\% fueron mujeres; edad promedio 70.8 años; $93 \%$ tenía conocimiento de los programas de donación de órganos y tejidos con fines de trasplante; $60.9 \%$ consideraba la posibilidad de donación de órganos con este fin; $50 \%$ mencionó tener conocimiento respecto a la donación de tejido cerebral con fines de investigación; $50 \%$ consideró esta posibilidad en un futuro $\left(\chi^{2}=0.348, \mathrm{gl}=1, \mathrm{p}>0.05\right)$. Conclusión: La intención de donación de cerebros en materia de investigación resulta escasa, en comparación con otros programas de donación de órganos para trasplante. La falta de difusión y la toma de conciencia respecto al tema son quizá las causas más importantes.
\end{abstract}

Palabras clave: Actitud, donación de cerebros, investigación, bioética.

\begin{abstract}
The aim of this study was to assess the intention of older adults towards the intention of brain donation in research. Material and methods: Descriptive, cross-sectional study, sample population of 51 subjects attending the Manuel Escontria Health Center, located in the San Ángel area. Results: Of the 46 subjects included in the study, $78.3 \%$ were women; average age 70.8 years; $93 \%$ were aware of organ and tissue donation programs for transplant purposes, $60.9 \%$ considered the possibility of organ donation for this purpose; $50 \%$ mentioned having knowledge regarding brain tissue donation for research purposes, $50 \%$ considered this possibility in the future $\left(\chi^{2}=0.348, g l=1, p>0.05\right)$. Conclusion: Research intention to donate brains is low compared to other organ donation programs for transplantation. Lack of dissemination and awareness of the issue are perhaps the most important causes.
\end{abstract}

Keywords: Attitude, brain donation, research, bioethics.

Citar como: González-Méndez M, Aguiñaga-Chiñas N, Cantú-Quintanilla G. Actitud ante la intención de donación de cerebros con fines de investigación en adultos mayores. Rev Mex Traspl. 2021; 10 (2): 41-45. https://dx.doi.org/10.35366/101409 


\section{INTRODUCCIÓN}

\section{Antecedentes}

El envejecimiento acelerado de la población representa un reto importante para el sector salud debido a la magnitud y repercusión en la vida de los pacientes y personas cercanos a ellos, por lo que este fenómeno requerirá de un modelo de atención específico para contender adecuadamente con las nuevas necesidades de la sociedad en materia de salud pública.

Se espera que la incidencia de enfermedades neurodegenerativas como las demencias se duplique cada 20 años a nivel mundial en proporción con el envejecimiento de la población. ${ }^{1}$ La enfermedad de Alzheimer (EA) es la causa más común de las enfermedades neurodegenerativas en personas mayores de 65 años y representa la cuarta causa de muerte en México después de las enfermedades del corazón, cáncer y diabetes. Se estima que su prevalencia es de $10 \%$ en personas mayores de 65 años y de $50 \%$ en adultos de más de 85 años. ${ }^{2}$

\section{Diagnóstico y tratamiento de} enfermedades neurodegenerativas

En la actualidad no se cuenta con un método diagnóstico precoz a pesar de que el diagnóstico clínico de la enfermedad es certero mediante el uso de pruebas estandarizadas que se combinan con la historia clínica y técnicas de imagen, el diagnóstico definitivo lo dicta el estudio histopatológico del cerebro postmortem. ${ }^{3}$ Ahí los hallazgo1s se caracterizan por la acumulación de placas neuríticas (depósitos extracelulares del péptido b-amiloide), marañas neurofibrilares (acumulaciones intracelulares) y pérdida de sinapsis. ${ }^{4}$

El diagnóstico de un síndrome demencial en etapas tempranas de la enfermedad evitará decretar «la sentencia» a perder las facultades mentales superiores de manera rápidamente progresiva, impactando en la capacidad de juicio y deliberación.

El uso de tejido cerebral humano es la principal estrategia para estudiar la base molecular y celular de los trastornos neuropsiquiátricos como es el caso de los síndromes demenciales. ${ }^{5}$ Sin embargo, la disponibilidad actual está lejos de ser suficiente. Los profesionales de la salud representan el enlace fundamental para fomentar la conciencia pública en el tema de donación de órganos.

El conocimiento que se ha publicado respecto a la donación de cerebros con fines de investigación se puede subdividir en categorías basadas en tres tipos de decisiones. La primera categoría ha evaluado la disposición de las familias al aprobar o negar el consentimiento de su paciente fallecido. «La consideración de los deseos de los fallecidos y la contribución a la investigación médica como factores motivadores clave para el consentimiento de la donación cerebral dentro de las familias». La segunda categoría se centra en la actitud de las personas sanas respecto a la donación de cerebro. Respecto a la donación de cerebro se han examinado los factores que influyen en el consentimiento de la autopsia y la donación de cerebro por parte de individuos sanos. "Ser voluntario puede basarse principalmente en la posibilidad de ayudar a otras personas en el futuro, ya sea a familiares o a la población en general». Finalmente, existe literatura escasa que se enfoca en pacientes con enfermedades neurodegenerativas. Kuhta y colaboradores (2011) examinaron a pacientes con trastornos del movimiento, fomentando el conocimiento médico y «ofreciendo la esperanza de una posible cura para aquellas personas que podrían ser diagnosticadas con este tipo de padecimientos en un futuro». ${ }^{6}$

\section{Banco nacional de cerebros}

La recolección de cerebros para estudiar enfermedades neurológicas y psiquiátricas comenzó al final del siglo $\mathrm{XIX}^{7}$ y la bancarización cerebral sistemática se inició en la década de 1960 en los Estados Unidos. ${ }^{8}$

México, como el resto del mundo, ha iniciado una campaña para desarrollar bancos de cerebros, los cuales funcionan como un centro de acopio de encéfalos, sangre y fluidos que permiten estudiar y analizar el proceso de las enfermedades neurodegenerativas en especial la enfermedad de Alzheimer. ${ }^{7}$

El tejido cerebral humano postmortem proporcionado a estos bancos resulta considerablemente valioso debido a que la mayoría de las enfermedades neurodegenerativas humanas están restringidas en su forma completa a los seres humanos.

\section{Difusión y toma de conciencia}

En México se está desarrollando una campaña de concientización para la donación de órganos, lo cual permitirá favorecer la investigación, por ende, el conocimiento de sus causas. Se espera que esto reditúe en expectativas terapéuticas para el tratamiento, 
mejora de la calidad de vida o prolongar la de otras personas como ocurre en la donación de órganos para trasplante. ${ }^{7}$

Sin embargo, el mayor problema es el desconocimiento de esta iniciativa de que se pueden donar órganos para investigación y que, con el tiempo, redituará en mejoras para la población. Es necesario superar las limitaciones impuestas por la desinformación y generar una cultura de donación a través de la difusión objetiva. ${ }^{9,10}$

\section{Principios bioéticos involucrados en la} intención de donación órganos ${ }^{11}$

El principio de autonomía: la obtención de órganos bajo el modelo de donación se arraiga en el principio de autonomía y debe respetarse cuando sobrevenga la muerte. Sin embargo, estos deseos postmortem sólo se llevarán a cabo si el individuo los dio a conocer de alguna forma durante su vida (petición expresa escrita, comentarios verbales con médicos, familiares, amistades, u otras formas). Esto implica que el individuo debe conocer lo que es posible hacer con su cuerpo cuando se le haya declarado muerto; situación que se ve influida por el nivel educativo de la sociedad.

El principio de responsabilidad: se define como la obligación de todos aquéllos que acceden a la ciencia y la tecnología a tener conciencia de sus propias acciones, las cuales deben ir acorde con el respeto a la vida humana y a su preservación.

Solidaridad: si todo ser humano tiene derecho a encontrar lo que necesita para su crecimiento y desarrollo, la solidaridad implica hacer nuestras las necesidades de quienes no tienen esos recursos para que puedan obtener los medios de subsistencia y los instrumentos de progreso personal.
Subsidiariedad: es una realidad social con notables diferencias de oportunidades, por este principio se busca que quién más sabe, puede o tiene, vea y atienda al que carece en cualquiera de esos ámbitos. Lo anterior no limita ni la iniciativa ni la responsabilidad de las personas y grupos sociales, sino más bien las valora, fomenta y acrecienta. Además de lo previamente señalado, consideramos fundamental establecer una corresponsabilidad del equipo tratante con la pareja donante-receptor y su entorno social.

Voluntariedad: debe garantizar que las personas decidan libremente someterse a un procedimiento, tratamiento médico o estudio clínico sin que su permiso haya sido obtenido por medio de coerción, persuasión o manipulación.

El objetivo del presente proyecto es identificar el conocimiento que poseen los adultos mayores respecto a los programas de donación de cerebro que se realiza en los bancos nacionales de cerebros con el fin de investigar enfermedades neurodegenerativas y neuropsiquiátricas. Asimismo comparar la relación que existe entre la intención de donación de órganos y tejidos con fines de trasplante y la donación de éstos con fines de investigación.

\section{MATERIAL Y MÉTODOS}

Estudio transversal, descriptivo y analítico. Inicialmente se seleccionaron 51 adultos mayores de 60 años quienes acuden con regularidad al Centro de Salud T-III Dr. Manuel Escontria, ubicado en el sur de la Ciudad de México, a quienes se les realizó una evaluación neuropsicológica. Después de la evaluación cinco casos fueron referidos a otras instituciones y excluidos del presente proyecto, manteniendo un total de 46 participantes para la muestra final (Figura 1).

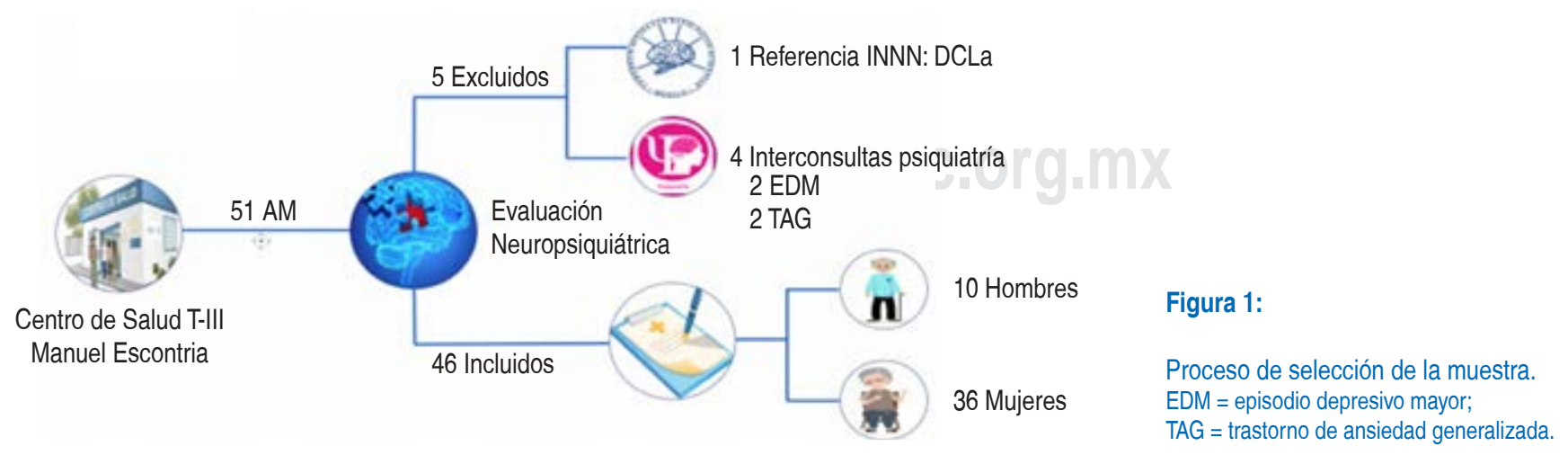




\section{Instrumentos}

La encuesta desarrollada incluyó reactivos que permitieron identificar de qué manera la cultura puede interferir en la toma de decisiones. La variable dependiente para evaluar la intención de donación de cerebro (una vez que el sujeto haya fallecido) se realizó a través de la pregunta directa, con opción de respuesta dicotómica (sí o no). Se realizó una evaluación clínica neuropsiquiátrica a través de la aplicación de escalas para descartar que los sujetos de estudio cumplieran criterios diagnósticos de depresión, ansiedad o alteraciones cognitivas que pudieran sesgar los resultados.

Además, se aplicó la escala de ansiedad ante la muerte de Templer, que es un cuestionario autoaplicable conformado por 15 ítems con respuestas tipo verdadero-falso. Los primeros nueve ítems tienen un valor de 1 si la respuesta es «verdadero», mientras que los últimos seis tienen un valor de 1 si la respuesta es «falso»; el puntaje mínimo a obtener es 0 y el máximo es 15 . El instrumento posee una consistencia interna de .86 para adultos mayores. ${ }^{12}$

\section{RESULTADOS}

Fueron 46 adultos mayores quienes cumplieron con los criterios de inclusión, la edad promedio de los participantes fue de $70.8 \pm 7.8$, la media de años de estudio fue de $9.3 \pm 5.03,78.3 \%$ fueron mujeres, $52.2 \%$ sin relación afectiva (de pareja), 69.6\% con diagnóstico de por lo menos una enfermedad crónico degenerativa, $89.1 \%$ reconoció pertenecer a una afiliación religiosa ( $100 \%$ definidos católicos).

Respecto al conocimiento e intención de donación de órganos y tejidos con fines de trasplante e intención de donación con el mismo fin, $93.5 \%$ de los sujetos encuestados $(n=43)$ afirmó tener conocimiento respecto a este tipo de programas y $60.9 \%(n=28)$ consideró la posibilidad postmortem.

De los sujetos encuestados, $52.2 \%(n=24)$ afirmó tener conocimiento respecto a los programas de donación de órganos y tejidos con fines de investigación y $54.3 \%(n=25)$ manifestó considerar la posibilidad de donar algún órgano o tejido para el estudio de padecimientos médicos (Tabla 1).

Respecto a la puntuación obtenida en la escala de Templer cuya puntuación mínima total es de 15 y la máxima total es de 60; la media de la puntuación en los sujetos de estudio fue de 22.39 con una $\mathrm{DE} \pm 5.32$; lo que indica que en general existe una mínima ansiedad ante la muerte (de acuerdo a las variables de miedo a la agonía, miedo a que la vida llegue a su fin y miedo a la muerte) en esta muestra poblacional.

Tabla 1: Comparación entre los sujetos que tienen o no intención de donar su cerebro con fines de investigación.

\begin{tabular}{|c|c|c|c|}
\hline & Desea donar & No desea donar & \\
\hline Características & $N=23$ & $N=23$ & p \\
\hline Edad en años & $69.9 \pm 7.1$ & $70.2 \pm 8.7$ & $0.88^{*}$ \\
\hline Años de estudio & $9.6 \pm 5.0$ & $9.1 \pm 5.0$ & $0.71^{*}$ \\
\hline Puntaje de Templer & $22.3 \pm 5.1$ & $22.5 \pm 5.6$ & $0.91^{*}$ \\
\hline Femeninos & $18(78.3)$ & $18(78.3)$ & $1.00^{\ddagger}$ \\
\hline No tiene pareja & $12(52.2)$ & $12(52.2)$ & $1.00^{\ddagger}$ \\
\hline \multicolumn{4}{|l|}{ Percepción de salud } \\
\hline Excelente & $1(4.3)$ & $2(8.7)$ & $0.65^{\S}$ \\
\hline Padece enfermedad crónica & $17(73.9)$ & $15(62.2)$ & $0.75^{\star}$ \\
\hline Afiliación religiosa & $22(95.7)$ & $19(82.6)$ & $0.34^{*}$ \\
\hline Tiene conocimiento del uso de donación de cerebros & $13(56.5)$ & $10(43.5)$ & $0.56^{*}$ \\
\hline
\end{tabular}

* Prueba t Student pareada, ${ }^{\ddagger}$ Prueba exacta de Fisher 2 colas, ${ }^{\S} \chi^{2}$ de Pearson, ${ }^{\natural} \chi^{2}$ lineal por lineal. 


\section{DISCUSIÓN}

Los resultados descritos revelan que la actitud de la población hacia la donación de órganos con fines de trasplante no son necesariamente las mismas que sus opiniones respecto a la donación de éstos con fines de investigación; quienes consideran la intención de donación de órganos y tejidos con fines de investigación (incluyendo tejido cerebral), en su mayoría también considerarían la posibilidad de donación con fines de trasplante.

Una explicación posible a esto es la falta de información y difusión en la población general respecto a la investigación médica y objetivos en los bancos nacionales de cerebros.

Los bancos de cerebros requieren de donantes, y como tal, llegar a inscribir a posibles candidatos es una parte esencial del éxito. A pesar de que existe evidencia de que acercarse a los posibles donadores favorece el reclutamiento, resulta importante que los bancos de cerebros publiciten sus estudios a nivel poblacional con un lenguaje que favorezca una adecuada comprensión y conocimiento respecto al funcionamiento y sus objetivos, asegurando así la inscripción de una cohorte de tamaño suficiente para satisfacer las necesidades de los diferentes tipos de estudio.

\section{CONCLUSIÓN}

Este estudio no pudo establecer diferencias significativas respecto a los factores que influyen en la intención de donación de tejido cerebral con fines de investigación. Sin embargo, el objetivo de esta investigación fue comprender la actitud que puede servir para futuras investigaciones.

Comprender la actitud y opiniones de la población con respecto al conocimiento de donación de tejido ce- rebral en materia de investigación tiene el potencial de mejorar la información que se debe proporcionar como parte de la difusión de los programas que existen en los biobancos.

\section{REFERENCIAS}

1. WHO. Dementia: a public health priority. Geneva: World Health Organization Alzheimer's Disease International. 2015.

2. ADI. World Alzheimer report. Alzheimer's Disease International. 2015.

3. Duyckaerts C. Diagnosis and staging of Alzheimer disease. Neurobiol Aging. 1997; 18: 33-42.

4. Ortíz G. Marcadores bioquímicos y moleculares en la enfermedad de Alzheimer. Gutiérrez L, editor: INGER; 2011.

5. Lesson G. Increasing longevity and the new demography of death. International Journal of Population Research. 2014.

6. Fonseca MK, Rodrigues-Neto E, Costa AS, Rockembach MA, Padilha RS, Fernandez LL et al. Assessing families' and patients' attitudes toward brain donation for research purposes in a Brazilian population sample. Cell Tissue Bank. 2015; 16: 287-294.

7. Albert M. The diagnosis of mild cognitive impairment due to Alzheimer's disease: Recommendations from the National Institute on Aging-Alzheimer's Association workgroups on diagnostic guidelines for Alzheimer's disease. Alzheimers Dement. 2011; 7 (3): 270-279.

8. Bauer K. Ethical issues in tissue banking for research: a brief review of existing organizational policies. Theor Med Bioeth. 2004; 25 (2): 113-142.

9. Moody C. Strategy for brain banking in the UK. J Neural Transm. 2008; 115: 1729 .

10. Sarris M. Banking for the future: an Australian experience in brain banking. Pathology. 2002; 34: 225-259.

11. Pérez R. La construcción de la bioética 2014.

12. Rivera-Ledesma A, Lena MML. Propiedades psicométricas de la escala de ansiedad ante la muerte de Templer en sujeto mexicanos. Diversitas. 2010; 6 (1): 135-140.

Correspondencia:

Dra. Marlette González-Méndez

Augusto Rodin Núm. 498,

Col. Insurgentes Mixcoac, 03920,

Alcaldía Benito Juárez, Ciudad de México, CDMX.

E-mail: dramarlette@gmail.com 